\title{
Belief Revision II: Ranking Theory
}

\author{
Franz Huber \\ Department of Philosophy \\ University of Toronto, Canada \\ franz.huber@utoronto.ca \\ http://huber.blogs.chass.utoronto.ca/ \\ penultimate version: please cite the paper in the \\ Philosophy Compass
}

July 7, 2014

\section{Contents}

1 Ranking Theory 3

2 Outlook 13 


\begin{abstract}
Belief revision theory studies how an ideal epistemic agent should revise her beliefs when she receives new information. In part I have first presented the AGM theory of belief revision (Alchourrón \& Gärdenfors \& Makinson 1985). Then I have focused on the problem of iterated belief revisions. In part II I will first present ranking theory (Spohn 1988). Then I will show how it solves the problem of iterated belief revisions. I will conclude by sketching two areas of future research.
\end{abstract}




\section{Ranking Theory}

Ranking functions have been introduced by Spohn (1988) to represent qualitative conditional belief. A most comprehensive overview can be found in Spohn (2012). The theory is quantitative or numerical in the sense that ranking functions assign numbers to sentences or propositions. These numbers are needed for the definition of conditional ranking functions representing conditional beliefs. As we will see, though, once conditional ranking functions are defined we can interpret everything in purely qualitative, but conditional terms.

Instead of taking the objects of belief to be sentences of a formal language it is both more general and more convenient to take them to be propositions of some field or algebra over a set of possible worlds $W$. A set of subsets of $W$, $\mathcal{A} \subseteq \wp(W)$, is an algebra over $W$ if and only if (i) the empty or contradictory set $\emptyset$ is a proposition in $\mathcal{A}$, (ii) the complement or negation of $A, W \backslash A=\bar{A}$, is a proposition in $\mathcal{A}$ if $A$ is a proposition in $\mathcal{A}$, and (iii) the union or disjunction of $A$ and $B, A \cup B$, is a proposition in $\mathcal{A}$ if both $A$ and $B$ are propositions in $\mathcal{A}$.

If we have a formal language $\mathcal{L}$ and $W_{\mathcal{L}}$ is the set of all models or truth-value assignments of $\mathcal{L}$, then $\mathcal{A}=\left\{\llbracket \alpha \rrbracket \subseteq W_{\mathcal{L}}: \alpha \in \mathcal{L}\right\}$ is an algebra of propositions over $W_{\mathcal{L}}$, where $\llbracket \alpha \rrbracket=\left\{w \in W_{\mathcal{L}}: w \models \alpha\right\}$ is the set of models of $\alpha$, or the set of truth-value assignments under which $\alpha$ is true. So for every formal language of sentences there is an algebra of propositions over the set of models of the formal language. Since the converse is not true, the semantic framework of propositions is more general than the syntactic framework of sentences (for details see Huber 2006).

As should become clear, it is also more convenient. Consider a set of possible worlds $W$ and an algebra of propositions $\mathcal{A}$ over $W$. A function $\varrho: \mathcal{A} \rightarrow \mathbb{N} \cup\{\infty\}$ from $\mathcal{A}$ into the set of natural numbers $\mathbb{N}$ extended by $\infty, \mathbb{N} \cup\{\infty\}$, is a ranking function on $\mathcal{A}$ just in case for any $A, B \in \mathcal{A}$ :

$$
\begin{aligned}
\varrho(W) & =0 \\
\varrho(\emptyset) & =\infty \\
\varrho(A \cup B) & =\min \{\varrho(A), \varrho(B)\}
\end{aligned}
$$

For a non-empty or consistent proposition $A \neq \emptyset$ from $\mathcal{A}$ the conditional ranking function $\varrho(\cdot \mid A): \mathcal{A} \backslash\{\emptyset\} \rightarrow \mathbb{N} \cup\{\infty\}$ based on the unconditional ranking function $\varrho(\cdot): \mathcal{A} \rightarrow \mathbb{N} \cup\{\infty\}$ is defined as

$$
\varrho(\cdot \mid A)=\left\{\begin{array}{lll}
\varrho(\cdot \cap A)-\varrho(A), & \text { if } & \varrho(A)<\infty, \\
0, & \text { if } & \varrho(A)=\infty .
\end{array}\right.
$$


Stipulating $\varrho(\emptyset \mid A)=\infty$ ensures that every conditional ranking function is a ranking function on $\mathcal{A}$. A ranking function $\varrho$ is regular if and only if $\varrho(A)<\varrho(\emptyset)$ for all non-empty or consistent propositions $A$ from $\mathcal{A}$. In contrast to probability theory it is always possible to define a regular ranking function, no matter how rich or fine-grained the underlying algebra of propositions.

Ranks are interpreted epistemically as grades of disbelief. A proposition $A$ is disbelieved conditional on a proposition $C$ just in case $A$ is assigned a positive rank conditional on $C, \varrho(A \mid C)>0$. It takes getting used to read positive numbers in this "negative" way, but mathematically this is the simplest formulation. Please note that it follows from the definition of conditional ranks that one should not disbelieve a proposition conditional on itself, $\varrho(A \mid A)=0$. In epistemic terms the first axiom says that one should not disbelieve the tautological proposition $W$. The second axiom says that one should disbelieve the empty or contradictory proposition $\emptyset$ with maximal strength $\infty$. Given the definition of conditional ranks, the second axiom can be read in purely qualitative, but conditional terms: it says that one should disbelieve the empty or contradictory proposition conditional on any proposition (with a finite rank).

Part of what the third axiom says is that one should disbelieve a disjunction $A \cup B$ just in case one disbelieves both its disjuncts $A$ and $B$. Given the definition of conditional ranks, the third axiom extends this requirement to conditional beliefs. As just noted we may assume one should not disbelieve a proposition conditional on itself. Given this the third axiom says - in purely qualitative, but conditional terms - the following: one should conditionally disbelieve a disjunction $A \cup B$ just in case one conditionally disbelieves both its disjuncts $A$ and $B$.

Epistemically interpreted axioms (1)-(3) are synchronic norms for organizing one's conditional beliefs at a given moment in time. They are supplemented by diachronic norms for updating one's beliefs over time if new information of various formats is received. The first update rule is defined for the case where the new information comes in form a certainty and mirrors the update rule of strict conditionalization from probability theory.

Update Rule 1 (Plain Conditionalization, Spohn 1988) If $\varrho(\cdot): \mathcal{A} \rightarrow \mathbb{N} \cup\{\infty\}$ is your ranking function at time $t$ and between $t$ and $t^{\prime}$ you become certain of $E \in \mathcal{A}$ and no logically stronger proposition (in the sense that $E$ is the logically strongest proposition whose negation is assigned $\infty$ as new rank at $\left.t^{\prime}\right)$, then your ranking function at time $t^{\prime}$ should be $\varrho_{E}(\cdot): \mathcal{A} \rightarrow \mathbb{N} \cup\{\infty\}, \varrho_{E}(\cdot)=\varrho(\cdot \mid E)$, where for all non-empty or consistent $B \in \mathcal{A}$ :

$$
\varrho_{E}(B)=\varrho(B \cap E)-\varrho(E) \text { and } \varrho(\emptyset \mid E)=\infty \text {. }
$$


The second update rule is defined for the case where the new information comes in form of new ranks for the elements of a partition and mirrors the update rule of Jeffrey conditionalization from probability theory (Jeffrey 1983).

Update Rule 2 (Spohn Conditionalization, Spohn 1988) If $\varrho(\cdot): \mathcal{A} \rightarrow \mathbb{N} \cup$ $\{\infty\}$ is your ranking function at time $t$ and between $t$ and $t^{\prime}$ your ranks on the evidential partition $\left\{E_{i} \in \mathcal{A}: i \in I\right\}$ change to $n_{i} \in \mathbb{N} \cup\{\infty\}$ with $\min \left\{n_{i}: i \in I\right\}=$ 0 , and $n_{i}=\infty$ if $\varrho\left(E_{i}\right)=\infty$, and your finite ranks change on no finer partition, then your ranking function at time $t^{\prime}$ should be $\varrho_{E_{i} \rightarrow n_{i}}(\cdot): \mathcal{A} \rightarrow \mathbb{N} \cup\{\infty\}$,

$$
\varrho_{E_{i} \rightarrow n_{i}}(\cdot)=\min _{i \in I}\left\{\varrho\left(\cdot \mid E_{i}\right)+n_{i}\right\} .
$$

The third update rule is defined for the case where the new information reports the differences between the old and the new ranks for the elements of a partition and mirrors the update rule of Field conditionalization from probability theory (Field 1978).

Update Rule 3 (Shenoy Conditionalization, Shenoy 1991) If $\varrho(\cdot): \mathcal{A} \rightarrow \mathbb{N} \cup$ $\{\infty\}$ is your ranking function at time $t$ and between $t$ and $t^{\prime}$ your ranks on the evidential partition $\left\{E_{i} \in \mathcal{A}: i \in I\right\}$ change by $z_{i} \in \mathbb{N}$, where $\min \left\{z_{i}: i \in I\right\}=0$, and your finite ranks change on no finer partition, then your ranking function at time $t^{\prime}$ should be $\varrho_{E_{i} \uparrow z_{i}}(\cdot): \mathcal{A} \rightarrow \mathbb{N} \cup\{\infty\}$,

$$
\varrho_{E_{i} \uparrow z_{i}}(\cdot)=\min _{i \in I}\left\{\varrho\left(\cdot \cap E_{i}\right)+z_{i}-m\right\}, \quad m=\min _{i \in I}\left\{z_{i}+\varrho\left(E_{i}\right)\right\} .
$$

Spohn conditionalizing $E$ and $\bar{E}$ to 0 and $n$, respectively, keeps the relative positions of all possible worlds in $E$ and all possible worlds in $\bar{E}$ fixed. It impoves the rank of $E$ to 0 and changes the rank of $\bar{E}$ to $n$. Shenoy conditionalizing $E$ and $\bar{E}$ by 0 and $n$, respectively, improves the possibilities within $E$ by $n$, as compared to the possibilities in $\bar{E}$. $m$ is a normalization parameter ensuring that at least one possible world is assigned rank zero so that the result is a ranking function. In both cases the new information consists of a (partition of) proposition(s) together with a (list of) number(s). This reflects the fact that the quality of new information varies with the reliability of its source: it makes a difference if the weather forecast predicts that it will rain, if a friend Sophia trusts tells her so, or if she sees herself that it is raining. In each case the proposition Sophia learns is that it is raining, but the effect of the new information on her old beliefs may be a different one in each case. The difference in the reliability of the sources of the new information is reflected in the number(s) accompanying the proposition(s). The effect the new information has on Sophia's old beliefs depends on those numbers. 
Sophia's ranking function $R$ will assign a positive rank to the proposition $\bar{A}$ that it will not be sunny on Wednesday. $R$ will assign a greater rank to the proposition $\bar{B}$ that it will not rain on Tuesday. And $R$ will assign an even greater rank to the proposition $\bar{C}$ that weather forecasts are not always reliable so that $0<R(\bar{A})<R(\bar{B})<R(\bar{C})$. More generally, the ordering $\alpha \leq_{R} \beta$ on $\mathcal{L}$ just in case $R(\overline{\llbracket \beta \rrbracket}) \leq R(\overline{\llbracket \alpha \rrbracket})$ is an entrenchment ordering for $\mathcal{B}=\{\gamma \in \mathcal{L}: R(\overline{\mathbb{\llbracket} \gamma \rrbracket})>0\}$. In other words, the set of propositions $\mathbf{S}_{R}=\left\{R^{-1}(n) \subseteq W: n \in \mathbb{N}\right\}$ is a system of spheres in $W$ centered on $R^{-1}(0)$, where $R^{-1}(n)=\{w \in W: R(\{w\})=n\}$ is the set of possible worlds that are assigned rank $n .{ }^{1}$ In still other words, the ordering $w \leq_{R} w^{\prime}$ on $W$ just in case $R(\{w\}) \leq R\left(\left\{w^{\prime}\right\}\right)$ is a plausibility ordering on $W$ with the center being the set of most plausible worlds, $\{v \in W: R(\{v\})=0\}=$ $\{v \in W: R(\{v\}) \leq R(\{w\}) \forall w \in W\}$. Therefore ranking theory covers AGM belief revision theory. It also covers the four additional postulates $* 9-* 12$ for iterated belief revision proposed by Darwiche \& Pearl (1997) and presented in part I, as can easily be verified by checking that the four postulates $\leq 5-\leq 8$ hold for $\leq_{S}$ (see also Spohn 2012: §5.6).

When Sophia learns on Monday that the weather forecast for Tuesday and Wednesday predicts rain, she has to tell us how strongly she now disbelieves the proposition $\bar{D}$ that the weather forecast for Tuesday and Wednesday does not predict rain in order for Spohn conditionalization to tell her how to revise her beliefs. As an approximation it suffices if she tells us how many information sources saying $\bar{D}$ it would now take for her to give up her disbelief in $\bar{D}$, as compared to how many information sources saying $\bar{X}$ it would then have taken for her to give up her disbelief that $\bar{X}$ for $X=A, B, C, D$. Suppose Sophia's old ranks are $R(\bar{A})=1$, $R(D)=2, R(\bar{B})=5$, and $R(\bar{C})=7$, and her new rank is $R^{*}(\bar{D})=13$. According to Spohn conditionalization Sophia's new ranks are:

$$
R^{*}(X)=\min \{R(X \mid D)+0, R(X \mid \bar{D})+13\}
$$

In order to calculate Sophia's new ranks $R^{*}(X)$ we thus have to know her old conditional ranks $R(X \mid D)$ and $R(X \mid \bar{D})$ as well as her new ranks for the conditions $D$ and $\bar{D}$. This in turn requires us to know her old ranks for various conjunctions. Suppose the numbers are as follows:

\footnotetext{
${ }^{1}$ We make the simplifying assumption that the algebra of propositions $\mathcal{A}$ contains all singletonpropositions $\{w\}$, for all possible worlds $w$ in $W$. If that assumption is not made, the system of spheres $\mathbf{S}_{R}$ and the plausibility ordering $\leq_{R}$ have to be defined in a slightly more complicated way.
} 
For R:

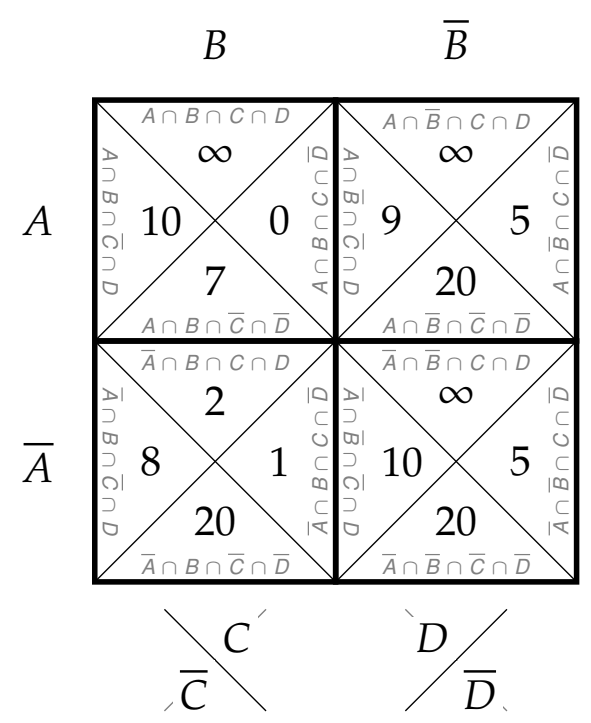

For $R^{*}$ :

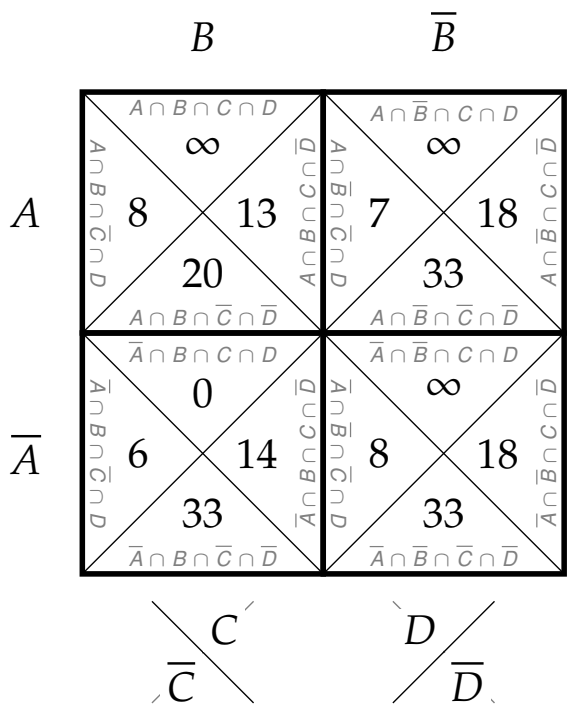

Then Sophia's new ranks are $R^{*}(\bar{C})=6, R^{*}(\bar{B})=7, R^{*}(A)=7, R^{*}(\bar{D})=13$. Note that $C$ is a proposition Sophia believes both before and after revision by $D, R(\bar{C})>0$ and $R^{*}(\bar{C})>0$, although $\bar{D}$ is positively relevant to, and so not independent of, $C$ in the sense that $R(\bar{C} \mid \bar{D})=7>6=R(\bar{C} \mid D)$. In other words, Sophia receives new information $D$ whose negation is positively relevant to, and so not independent of, her belief that $C$ without making her give up her belief that $C$. On the other hand, if Sophia considers $\bar{D}$ independent of a proposition $X$ before revision by $D$, then she also does so after revision by $D$. More generally, if two propositions $A$ and $B$ are independent according to a ranking function $R$, $R(A \mid B)=R(A \mid \bar{B})$ and $R(\bar{A} \mid B)=R(\bar{A} \mid \bar{B})$, then $A$ and $B$ are independent according to any ranking function $R^{*}$ that results from $R$ by what we may call a "Spohn shift" on the partition $\{B, \bar{B}\}$, i.e. the result of Spohn conditionalization on that partition for an arbitrary pair of numbers. This feature, which is known as rigidity, vindicates the idea behind Jin \& Thielscher (2007)'s proposal to preserve independencies by fixing their notion of independence. ${ }^{2}$

\footnotetext{
${ }^{2}$ While rigidity is generally considered to be a desirable feature of an update rule, it should be noted that Weisberg (2009; ms) uses rigidity to argue that neither Bayesianism nor ranking theory can handle a phenomenon he terms perceptual undermining.
} 
Spohn conditionalization gives Sophia a complete new ranking function $R^{*}$ that she can use to revise her newly acquired belief set $\mathcal{B}^{*}=\left\{X \in \mathcal{A}: R^{*}(\bar{X})>0\right\}$ a second time when she learns on Tuesday that it is sunny after all. All she has to do is tell us how strongly she then disbelieves the proposition $B$ that it will rain on Tuesday. If $R^{* *}(B)=13$, Sophia's new ranks are $R^{* *}(\bar{A})=1, R^{* *}(C)=11$, $R^{* *}(\bar{D})=11$.

For $R^{*}$ :

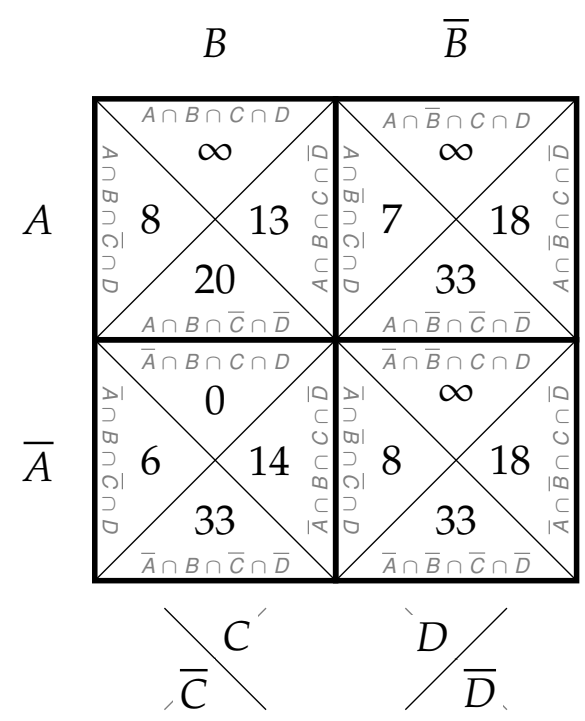

For $R^{* *}$ :

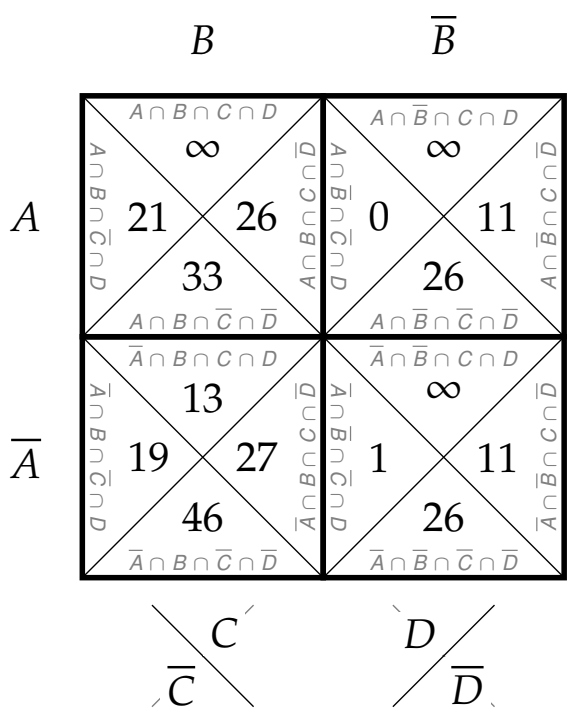

This means that Sophia did not mishear the weather forecast, but was too gullible, say, and so has to give up her belief $C$ that weather forecasts are always reliable. In addition she also has to regain her belief $A$ that it will be sunny on Wednesday. This way ranking theory handles indefinitely iterated belief revisions.

We can picture Sophia's doxastic career as a sequence of "onions", except that now the layers carry numbers reflecting how far apart they are from each other. A different way to picture the situation is to allow for empty layers and to have one, possibly empty, layer for each natural number $n$. See below.

Ranking theory is a normative theory that addresses the question how an ideal epistemic agent should revise her beliefs if she receives new information of various formats. But why should an ideal epistemic agent obey the norms of ranking theory? That is, why should an ideal epistemic agent organize her conditional 
beliefs at a given moment in time according to axioms (1)-(3)? And why should she update her beliefs over time according to update rules (1)-(3) if she receives new information of the appropriate format? To answer these questions we need a bit of terminology.

An agent's degree of entrenchment for a proposition $A$ is defined as the number of independent and minimally positively reliable information sources saying $A$ that it takes for the agent to give up her disbelief in $A$. Independent and minimally positively reliable information sources for a proposition are idealized entities that are used to define an agent's degrees of entrenchment. Information sources as we know them usually are neither independent nor minimally positively reliable.

The agent's grades of disbelief, her ranks, are theoretical entities. Their relation to the agent's idealized, but otherwise observable degrees of entrenchment is a delicate one: minimally degrees of entrenchment are used to measure grades of disbelief; maximally the latter can be defined in terms of the former. ${ }^{3}$

Let $\varrho$ be the agent's entrenchment function, i.e. the function that summarizes her degrees of entrenchment for all the propositions under consideration. Then the agent's belief set $\mathcal{B}_{\varrho}$ is defined as the set of propositions whose negations have a positive degree of entrenchment, $\mathcal{B}_{\varrho}=\{A \in \mathcal{A}: \varrho(\bar{A})>0\}$. An agent's belief set $\mathcal{B}_{\varrho} \subseteq \mathcal{A}$ is consistent if and only if for every finite set of propositions $\mathcal{B} \subseteq \mathcal{B}_{\varrho}$ : $\cap \mathcal{B} \neq \emptyset$. An agent's belief set $\mathcal{B} \subseteq \mathcal{A}$ is deductively closed if and only if for every finite set of propositions $\mathcal{B} \subseteq \mathcal{B}_{\varrho}$ and all propositions $A \in \mathcal{A}$ : if $\cap \mathcal{B} \subseteq A$, then $A \in \mathcal{B}_{\varrho}$.

Now we can answer the question why an ideal epistemic agent should organize her conditional beliefs at a given moment in time according to axioms (1)-(3), and why she should update her beliefs over time according to update rules (1)-(3) if she receives new information of the appropriate format. She should do so, because

Theorem 1 An agent's belief set $\mathcal{B}_{\varrho}$ based on her entrenchment function $\varrho$ is and will always be consistent and deductively closed, possibly conditional on some element of the evidential partition, if and only if $\varrho$ is a ranking function and, depending on the format of the information received, the agent updates according to update rule 1,2 , or 3 .

For a proof of theorem 1 the reader is referred to Huber (2007).

\footnotetext{
${ }^{3}$ The situation is somewhat similar in Bayesianism, where betting ratios or fair betting ratios minimally are used to measure degrees of belief, and maximally are used to define them (Eriksson \& Hájek 2007). Whatever the exact relation between (fair) betting ratios and degrees of belief, the former are the central notion in the best known argument in favor of Bayesianism, the Dutch Book Argument (Easwaran 2011a).
} 
This argument is supposed to establish the thesis that an agent's beliefs should obey the synchronic and diachronic rules of the ranking calculus. The argument provides a means-ends justification for this thesis in the spirit of epistemic consequentialism (Percival 2002, Stalnaker 2002). The idea is that obeying the normative constraints of the ranking calculus is a (necessary and sufficient) means to attaining the end of being "eternally consistent and deductively closed." The latter end in turn is a (necessary, but insufficient) means to attaining the end of always having only true beliefs, and as many as possible thereof. Brössel \& Eder \& Huber (forthcoming) discuss the importance of this result as well as its Bayesian role-model, Joyce's (1998; 2009) "non-pragmatic vindication of probabilism", for considering epistemic rationality a form of instrumental rationality and for meansends epistemology in general. Alternatively one may use the representation result by Hild \& Spohn (2008) or the rank-theoretic decision theory by Giang \& Shenoy (2000) to obtain a justification of ranking theory that is more deontological in spirit.

Sophia's ranking functions $R$ and $R^{*}$ can be pictured as "numbered onions."

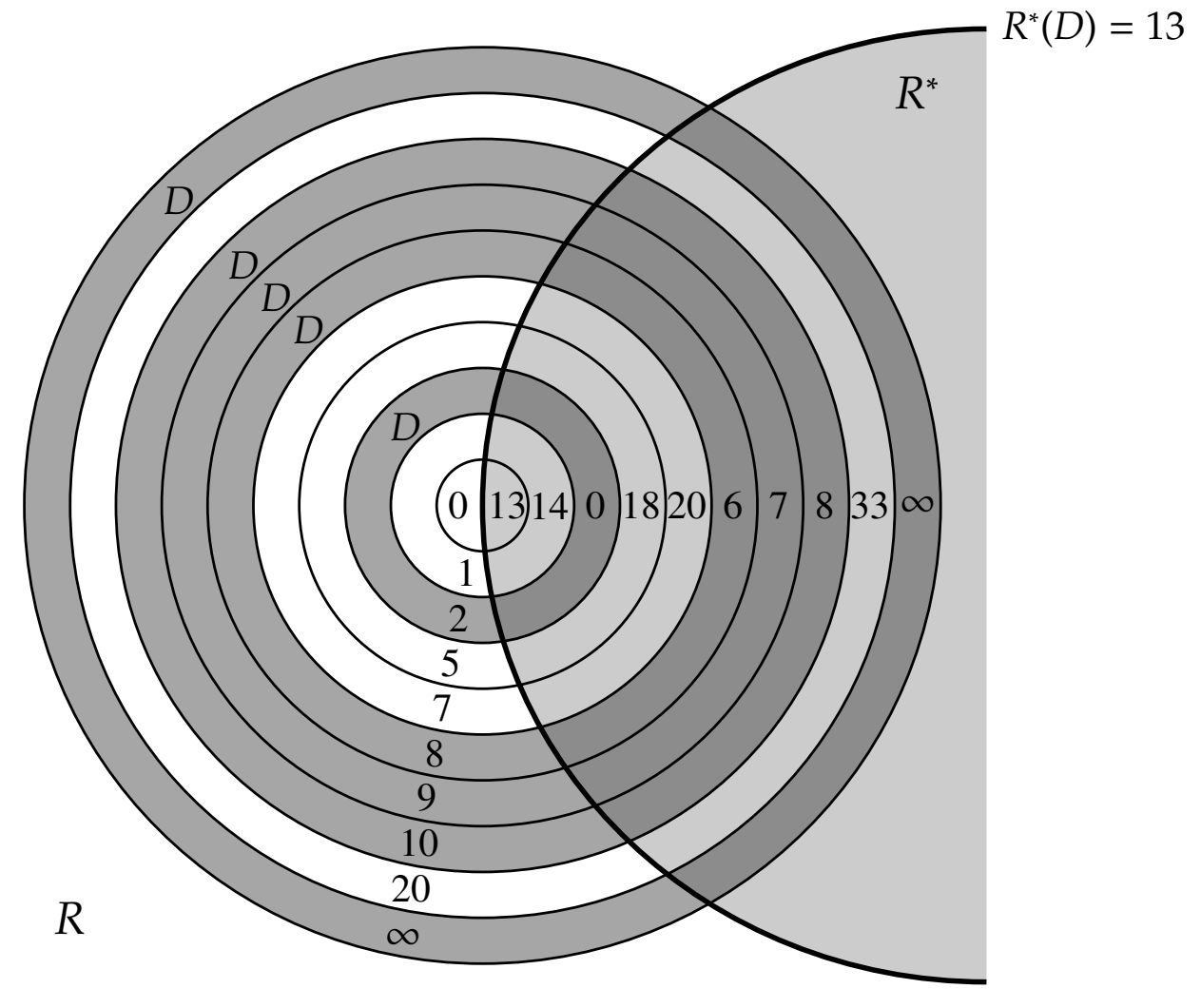


Alternatively Sophia's ranking function $R$ can be pictured as a numbered onion with one, possibly empty, layer $R^{-1}(n)$ for each natural number $n$. Sophia's old rank for $D$ is $2, R(D)=2$, and her old rank for $\bar{D}$ is $0, R(\bar{D})=0$. Sophia's new ranking function $R^{*}$ arises from her old ranking function $R$ by first improving the $D$-worlds by 2 ranks so that the new rank of $D$ is $0, R^{*}(D)=0$. In a second step the $\bar{D}$-worlds are deteriorated by 13 ranks so that the new rank of $\bar{D}$ is 13 , $R^{*}(\bar{D})=13$. The relative positions of the $D$-worlds and the $\bar{D}$-worlds expressed in the conditional ranking functions $R(\cdot \mid D)=R^{*}(\cdot \mid D)$ and $R(\cdot \mid \bar{D})=R^{*}(\cdot \mid \bar{D})$ are kept fixed. 


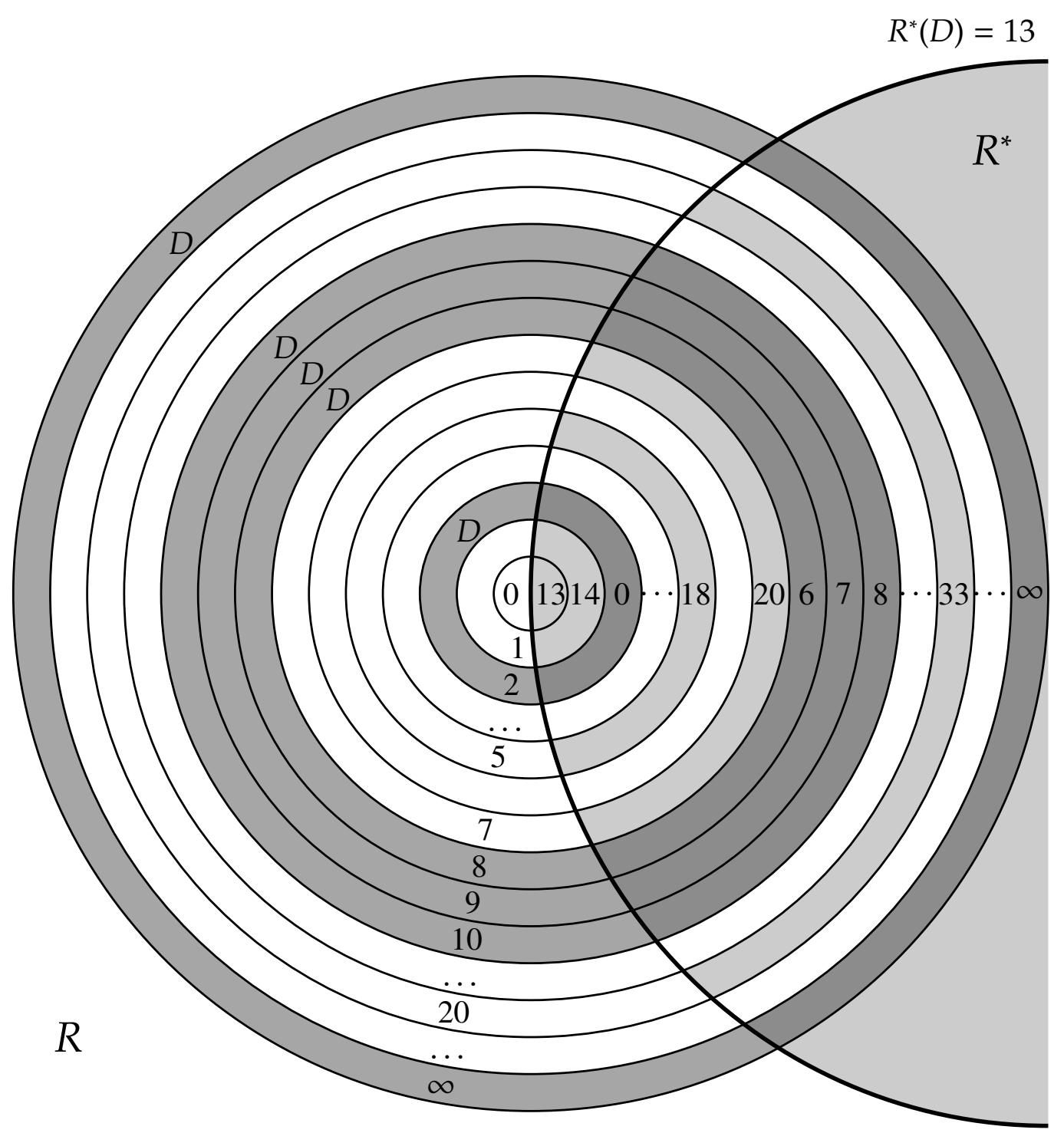




\section{Outlook}

Belief revision theory studies how an ideal epistemic agent should revise her beliefs when she receives new information. In the present two articles I have restricted the discussion to the case where the new information comes in the qualitative form of a sentence or proposition of the agent's language or algebra, as in the AGM theory of belief revision; or in the comparative form of the relative positions of an input sentence and a reference sentence, as in two-dimensional belief revision; or in the quantitative form of new degrees of (dis)belief for various propositions, as in the case of ranking theory. Let us call information that concerns only individual sentences or propositions of the agent's language or algebra factual information, and the corresponding changes in belief factual changes.

There are at least two other forms of information an ideal epistemic agent can receive and, corresponding to these, at least two forms of non-factual change. The first of these non-factual changes takes place when the agent learns that her language or algebra was too poor or coarse-grained. For instance, the agent may start out with a language that allows her to distinguish between red wine and white wine, and then may acquire the concept of rosé. Or she may learn that among the red wines one can distinguish between barriques and non-barriques. When the agent receives such conceptual information she should perform a conceptual change. A prominent conceptual change is that of logical learning. In the syntactic AGM framework logical learning is normally studied in terms of belief bases (Hansson 1999). Belief bases differ from belief sets by not being required to be closed under the logical consequence relation. Huber (2009: 23f) sketches how logical learning and conceptual changes in general can be dealt with in the semantic framework of ranking theory.

The other form of non-factual information is meta-information, and agents receiving meta-information should perform a meta-change (Stalnaker 2009). Information about (in)dependencies among propositions, as reported in causal claims or claims about counterfactual or indicative conditionals, is a prominent form of meta-information. I think the challenge raised by Weisberg (ms) is a plea for update rules that allow the ideal epistemic agent to learn about (in)dependencies. In the syntactic AGM framework one might be able to study meta-changes with the help of dynamic doxastic logic (Segerberg 1995, Lindström \& Rabinowicz 1999), which allows one to reason about one's own beliefs (this is known as autoepistemology). In the semantic framework of ranking theory auto-epistemology has been studied by Spohn (2012: §9) based on Hild (1998). It remains to be seen whether all meta-changes can be understood in an auto-epistemological way. 


\section{Acknowledgments}

I am grateful to Benjamin Bewersdorf, Jennifer Nagel, Hans Rott, Sherrilyn Roush, Wolfgang Spohn, Jonathan Weisberg, and an anonymous referee for helpful comments on earlier versions of these two papers. Robert Lehnert helped me with the design of the graphics. My research was in part supported by the German Research Foundation through its Emmy Noether program.

\section{Short Biography}

Franz Huber is an assistant professor in the Department of Philosophy at the University of Toronto. His research interests are in formal epistemology, general philosophy of science, and philosophical logic.

\section{References}

Alchourrón, Carlos E. \& Gärdenfors, Peter \& Makinson, David (1985), On the Logic of Theory Change: Partial Meet Contraction and Revision Functions. Journal of Symbolic Logic 50, 510-530.

Brössel, P. \& Eder, A. \& Huber, F. (forthcoming), Evidential Support and Instrumental Rationality. Philosophy and Phenomenological Research. DOI: 10.1111/j.1933-1592.2011.00543.x

Darwiche, Adnan \& Pearl, Judea (1997), On the Logic of Iterated Belief Revision. Artificial Intelligence 89, 1-29.

Easwaran, Kenny (2011a): Bayesianism I: Introduction and Arguments in Favor. Philosophy Compass 6, 312-320.

Eriksson, Lina \& Hájek, Alan (2007), What Are Degrees of Belief? Studia Logica 86, 185-215.

Field, Hartry (1978), A Note on Jeffrey Conditionalization. Philosophy of Science 45, 361-367.

Giang, Phan H. \& Shenoy, Prakash P. (2000), A Qualitative Linear Utility Theory for Spohn's Theory of Epistemic Beliefs. In C. Boutilier \& M. Goldszmidt (eds.), 
Uncertainty in Artificial Intelligence 16. San Francisco: Morgan Kaufmann, 220229.

Hansson, Sven O. (1999), A Textbook of Belief Dynamics. Theory Change and Database Updating. Dordrecht: Kluwer.

Hild, Matthias (1998), Auto-Epistemology and Updating, Philosophical Studies 92, 321-361.

Hild, Matthias \& Spohn, Wolfgang (2008), The Measurement of Ranks and the Laws of Iterated Contraction. Artificial Intelligence 172, 1195-1218.

Huber, Franz (2006), Ranking Functions and Rankings on Languages. Artificial Intelligence 170, 462-471.

Huber, Franz (2007), The Consistency Argument for Ranking Functions. Studia Logica 86, 299-329.

Huber, Franz (2009), Belief and Degrees of Belief. In F. Huber \& C. SchmidtPetri (eds.), Degrees of Belief. Synthese Library 342. Dordrecht: Springer, 1-33.

Jeffrey, Richard C. (1983), The Logic of Decision. 2nd ed. Chicago: University of Chicago Press.

Jin, Yi \& Thielscher, Michael 2007), Iterated Belief Revision, Revised. Artificial Intelligence 171, 1-18.

Joyce, James M. (1998), A Non-Pragmatic Vindication of Probabilism. Philosophy of Science 65, 575-603.

Joyce, James M. (2009), Accuracy and Coherence: Prospects for an Alethic Epistemology of Partial Belief. In F. Huber \& C. Schmidt-Petri (eds.), Degrees of Belief. Synthese Library 342. Dordrecht: Springer, 263-297.

Lindström, Sten \& Rabinowicz, Wlodek (1999), DDL Unlimited: Dynamic Doxastic Logic for Introspective Agents, Erkenntnis 50, 353-385.

Percival, Philip (2002), Epistemic Consequentialism. Proceedings of the Aristotelian Society, Supplementary Volume 76, 121-151.

Segerberg, Krister (1995), Belief Revision from the Point of View of Doxastic Logic. Bulletin of the IGPL 3, 535-553. 
Shenoy, Prakash P. (1991), On Spohn's Rule for Revision of Beliefs. International Journal of Approximate Reasoning 5, 149-181.

Spohn, Wolfgang (1988), Ordinal Conditional Functions: A Dynamic Theory of Epistemic States. In W.L. Harper \& B. Skyrms (eds.), Causation in Decision, Belief Change, and Statistics II. Dordrecht: Kluwer, 105-134.

Spohn, Wolfgang (2012), The Laws of Belief. Ranking Theory and its Philosophical Applications. Oxford: Oxford University Press.

Stalnaker, Robert C. (2002), Epistemic Consequentialism. Proceedings of the Aristotelian Society, Supplementary Volume 76, 153-168.

Stalnaker, Robert C. (2009), Iterated Belief Revision. Erkenntnis 70, 189-209.

Weisberg, Jonathan (2009), Commutativity or Holism? A Dilemma for Conditionalizers. British Journal for the Philosophy of Science 60, 793-812.

Weisberg, Jonathan (ms), Updating, Undermining, and Independence. 